\title{
Erratum
}

\section{Efficient One-Pot Synthesis of Substituted 1-Acylaminocyclohex-2-enes}

Sandra Hübner, Helfried Neumann, Axel Jacobi Wangelin, Stefan Klaus, Dirk Strübing, Holger Klein, Matthias Beller* Synthesis 2005, 2084.

The name of the third author was incorrectly represented. The correct name is Axel Jacobi von Wangelin. 